\title{
BOOLEAN ALGEBRAS OF PROJECTIONS
}

\author{
by P. G. SPAIN \\ (Received 16th January 1974)
}

Bade, in (1), studied Boolean algebras of projections on Banach spaces and showed that a $\sigma$-complete Boolean algebra of projections on a Banach space enjoys properties formally similar to those of a Boolean algebra of projections on Hilbert space. (His exposition is reproduced in (7: XVII).) Edwards and Ionescu Tulcea showed that the weakly closed algebra generated by a $\sigma$-complete Boolean algebra of projections can be represented as a von Neumann algebra; and that the representation isomorphism can be chosen to be norm, weakly, and strongly bicontinuous on bounded sets (8): Bade's results were then seen to follow from their Hilbert space counterparts. I show here that it is natural to relax the condition of $\sigma$-completeness to weak relative compactness; indeed, a Boolean algebra of projections has a $\sigma$-completion if and only if it is weakly relatively compact (Theorem 1). Then, following the derivation of the theorem of Edwards and Ionescu Tulcea from the Vidav characterisation of abstract $C^{*}$-algebras (see (9)), I give a result (Theorem 2) which, with its corollary, includes (1: $2.7,2.8,2.9,2.10,3.2,3.3,4.5)$.

Let $X$ be a complex Banach space with dual $X^{\prime}$; let $L(X)$ be the Banach algebra of (bounded linear) operators on $X$. Two weak topologies are used in this paper; the weak topology $\sigma\left(X, X^{\prime}\right)$ on $X$, and the weak (operator) topology on $L(X)$. Note that a subset $E$ of $L(X)$ is weakly relatively compact if and only if $E x$ is weakly relatively compact (in $X$ ) for each $x$ in $X$ (7: VI.9.2). The strong (operator) topology on $L(X)$ will also be used.

A projection on $X$ is an idempotent in $L(X)$. If $E$ is a projection, so is its complement $I-E$. If $E$ and $F$ are commuting projections, they have a least upper bound $E \vee F(=E+F-E F)$ and a greatest lower bound $E \wedge F(=E F)$. A set of commuting projections is a Boolean algebra of projections if it contains 0 and $I$, and is closed under complementation and the operations $\vee$ and $\wedge$.

A Boolean algebra of projections $B$ on $X$ is complete ( $\sigma$-complete) if for each subset (subsequence) $\left\{B_{\lambda}\right\}$ of $B$ there is a direct sum decomposition $X=Y \oplus Z$, where $Y$ is the closed subspace spanned by $\left\{B_{2} X\right\}, Z$ is the closed subspace $\bigcap\left(I-B_{\lambda}\right) X$, and the projection $B$ onto $Y$ along $Z$ belongs to $B$; then $B=\bigvee B_{\lambda}$. If $B$ is complete ( $\sigma$-complete) in this sense, then $B$ is complete ( $\sigma$-complete) as an abstract Boolean algebra.

If $B$ is $\sigma$-complete (even only as an abstract Boolean algebra), then $B$ is bounded (that is, there is a number $M$ with $\|B\| \leqq M$ when $B \in B$ (1: Theorem 2.2)). 
If $\boldsymbol{B}$ is complete ( $\sigma$-complete) and $\left(B_{\lambda}\right)$ is an increasing net (sequence) in $\boldsymbol{B}$, then $\bigvee B_{\lambda}=\lim B_{\lambda}$ in the strong topology (1: Lemma 2.3).

Similarly, if $\boldsymbol{B}$ is weakly relatively compact, then $\boldsymbol{B}$ is bounded and $\boldsymbol{B} x$ is weakly relatively compact for each $x$ in $X$. If $\left(B_{\lambda}\right)$ is an increasing net in $B$, then, by (2: Corollary 1), $\bigvee B_{\lambda}$ exists and $\bigvee B_{\lambda}=\lim B_{\lambda}$ in the strong topology.

Let $\Lambda$ be the Stone representation space of $B$. Write $K$ for the set of openand-closed subsets of $\Lambda, S$ for the set of Baire subsets of $\Lambda$. Let us write the representation map $K \rightarrow B$ in the form $\tau \mapsto B(\tau)$. Because $\Lambda$ is a totally disconnected compact Hausdorff space, $S$ is the $\sigma$-algebra (alternatively, the monotone class) generated by $K$; also, $L$, the linear (which is also the algebra) span of the characteristic functions of sets in $K$, is norm-dense in $C(\Lambda)$, the algebra of continuous functions on $\Lambda$.

Theorem 1. Let $B$ be a Boolean algebra of projections on a Banach space. Then $\boldsymbol{B}$ is weakly relatively compact if and only if $\boldsymbol{B}$ has a $\sigma$-completion.

Proof. Let $B$ be weakly relatively compact. Consider a sequence $\left(\tau_{n}\right)$ in $K$. By a remark above, $\left(B\left(\bigcup_{1}^{n} \tau_{k}\right)\right)$ increases, and converges strongly, to a projection in $\boldsymbol{B}^{s}$, the strong closure of $\boldsymbol{B}$. Countable iteration of this process will give a projection $\widetilde{B}(\tau)$ for each $\tau$ in $S$. It is easy to see that $\widetilde{B}: S \rightarrow B^{s}$ extends $B$, that $\widetilde{B}$ is a spectral measure and that $\widetilde{B}(S)$ is the $\sigma$-completion of $B$.

Conversely, assume that $B$ has a $\sigma$-completion $\tilde{B}$. Then, as in the preceding paragraph, $B: K \rightarrow B$ has an extension $\widetilde{B}: S \rightarrow \widetilde{B}$, showing that $\widetilde{B}$ is the range of a spectral measure. (This observation was of prime importance in (1).) Now the range of a vector measure is weakly relatively compact (3: Theorem 2.9 ), so $\tilde{\boldsymbol{B}} x$ is weakly relatively compact for each $x$ in $X$. Thus $\widetilde{B}$ is weakly relatively compact; whence $B$ is.

Theorem 2. Let $\boldsymbol{B}$ be a Boolean algebra of projections on a Banach space $X$. Suppose that $\boldsymbol{B}$ has a $\sigma$-completion (or equivalently, that $\boldsymbol{B}$ is weakly relatively compact). Then $\boldsymbol{B}$ has a completion contained in $\boldsymbol{B}^{s}$ (which is a complete Boolean algebra of projections), and the weak and strong topologies agree on $B^{s}$. Let $A$ be the norm-closed algebra generated by $B$; let $A^{w}$ be the weak closure of $A$. Then $B$ is complete if and only if $B=B^{s}$, if and only if $A=A^{w}$.

Proof. $X$ has an equivalent norm for which the members of $B$ are hermitian (in that they have real numerical range (see (6)) (4: Lemmas 2.2, 2.3). We may assume that $X$ has this norm. By (5: Theorem 2.1), the map $B: K \rightarrow B$ has an isometric linear extension to a map $L \rightarrow A$; this extension is an algebra homomorphism. Therefore $B: \boldsymbol{K} \rightarrow \boldsymbol{B}$ extends to an isometric algebra isomorphism $\tilde{\tilde{B}}: C(\Lambda) \rightarrow A ;$ and $\tilde{B}(f)=\int_{\Lambda} f d \tilde{B}(f \in C(\Lambda))$. So, in the terminology of (9), $A$ is representable by a spectral measure. By (9: Theorem 2), $A^{w}$ is a $W^{*}$. algebra; moreover, there are a Hilbert space $H$, a von Neumann algebra $\tilde{A}$ on 
$H$, and a $C^{*}$-isomorphism $A^{\mathrm{w}} \rightarrow \tilde{A}$ which is weakly and strongly bicontinuous on bounded sets. The theorem now follows from the corresponding Hilbert space results.

Corollary. Let $B$ be a bounded Boolean algebra of projections on a weakly complete Banach space $X$. Then $B$ satisfies the hypotheses of the theorem.

Proof. The map $\widetilde{B}: C(\Lambda) \rightarrow A$ may be defined as in the proof of the theorem. Then $C(\Lambda) \rightarrow X: f \mapsto B(f) x$ is weakly compact (3: Theorem 3.2), whence $B x$ is weakly relatively compact (for each $x$ in $X$ ); so $B$ is weakly relatively compact.

\section{Acknowledgement}

I first met the matters discussed here when a research student under the supervision of $\operatorname{Dr} \mathrm{H}$. R. Dowson: I thank him for his advice then and since.

\section{REFERENCES}

(1) W. G. BADE, On Boolean algebras of projections and algebras of operators, Trans. Amer. Math. Soc. 80 (1955), 345-360.

(2) J. Y. Barry, On the convergence of ordered sets of projections, Proc. Amer. Math. Soc. 5 (1954), 313-314.

(3) R. G. Bartle, N. Dunford and J. T. Schwartz, Weak compactness and vector measures, Canadian J. Math. 7 (1955), 289-305.

(4) E. Berkson, A characterization of scalar type operators on reflexive Banach spaces, Pacific J. Math. 13 (1963), 365-373. 1-8.

(5) E. Berkson, Some characterizations of C*-algebras, Illinois J. Math. 10 (1966),

(6) F. F. Bonsall and J. DunCan, Numerical Ranges of Operators on Normed Spaces and of Elements of Normed Algebras (Cambridge University Press, 1971). 1958).

(7) N. Dunford and J. T. SchwarTz, Linear Operators (Interscience, New York,

(8) D. A. EDWARds and C. T. IonesCu TulceA, Some remarks on commutative algebras of operators on Banach spaces, Trans. Amer. Math. Soc. 93 (1959), 541-551. 134.

(9) P. G. Spain, On commutative V*-algebras II, Glasgow Math. J. 13 (1972), 129-

\section{UNIVERSITY OF GLASGOW \\ GLASGOW G12 8QW}

